\title{
Left atrial structure and functional quantitation using cardiac magnetic resonance: comparison of manual delineation vs. multimodality tissue tracking based semi-automated methods
}

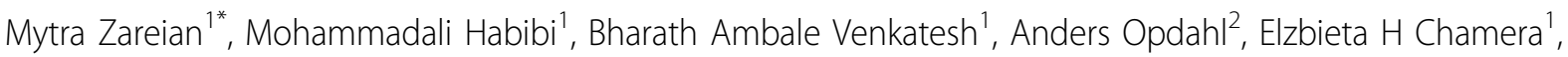 \\ Colin Wu ${ }^{3}$, Filip Zemrak', David Bluemke ${ }^{3}$, Joao A Lima ${ }^{1}$
}

From 17th Annual SCMR Scientific Sessions

New Orleans, LA, USA. 16-19 January 2014

\section{Background}

Left atrium (LA) volume and function are important markers of cardiovascular disease. LA volume can be assessed by several different methods. In clinical practice, the Simpson's method is well accepted as a reference standard, although there is no standardization for LA volume calculations. We aimed to compare the estimations of LA volume by the Simpson's method and the modified biplane Simpson's method; and to introduce Multimodality Tissue Tracking (MTT, Japan, Toshiba) as a new semi-automated method for quantifying LA function based on tissue feature tracking.

\section{Methods}

Thirty subjects (mean age: $71.3 \pm 8.7,87 \%$ male) including twenty subjects with cardiovascular events (4 atrial fibrillation, 18 myocardial scar from late gadolinium enhancement, 2 heart failure) and ten healthy subjects, with CMR imaging were evaluated in the Multi-Ethnic Study of Atherosclerosis (MESA). LA volumes were measured using the modified biplane Simpson's method from 2- and 4-chamber projections and the original Simpson's method using short-axis slices. For the manual methods, LA endo- and epicardial boundaries were delineated at left-ventricular end-diastole (Vmin), end-systole (Vmax) and just before the pre-atrial contraction (VpreA). Using MTT, LA endocardial and epicardial borders were manually delineated at end-systole and the boundaries were propagated automatically throughout the cardiac

${ }^{1}$ Cardiovascular Imaging, Johns Hopkins University, Baltimore, Maryland, USA Full list of author information is available at the end of the article cycle. LA total $(\mathrm{LAEF}=\mathrm{Vmax}-\mathrm{Vmin} / \mathrm{Vmax})$, active $($ LAAEF $=$ VpreA-Vmin $/$ VpreA $)$ and passive ejection fraction (LAPEF $=$ Vmax-VpreA/Vmax) were calculated (Figure 1). A two-tailed paired sample t-test is used to determine significant differences between two types of computations (Simpson's from short-axis images vs. Biplane Simpsons from long-axis images) and methods (MTT vs. manual). Pearson's correlation and BlandAltman analysis are used to examine the relationship between the two computations and methods. In addition, intra-class correlation coefficients (ICC) for inter and intra reader reproducibility are calculated.

\section{Results}

LA parameter analysis using the two different computations was significantly different for functional LA parameters by both methods (Table 1). LA functional parameters did not differ between manual and semi-automated methods (Table 1). LA volumes obtained from the Simpson's method was significantly different between manual and MTT methods (Table 1). Image analysis was less time consuming on average with MTT (Simpson's: MTT vs. manual: 3:10 min vs. 7:23 min; Biplane, MTT vs. manual: 1:30 min vs. 8:28 min). All parameters showed good to excellent intra and inter reader reproducibility (ICC; 0.69-0.99).

\section{Conclusions}

MTT derived biplane LA structure and function is accurate, less time consuming, highly reproducible and could potentially be used in large studies. 


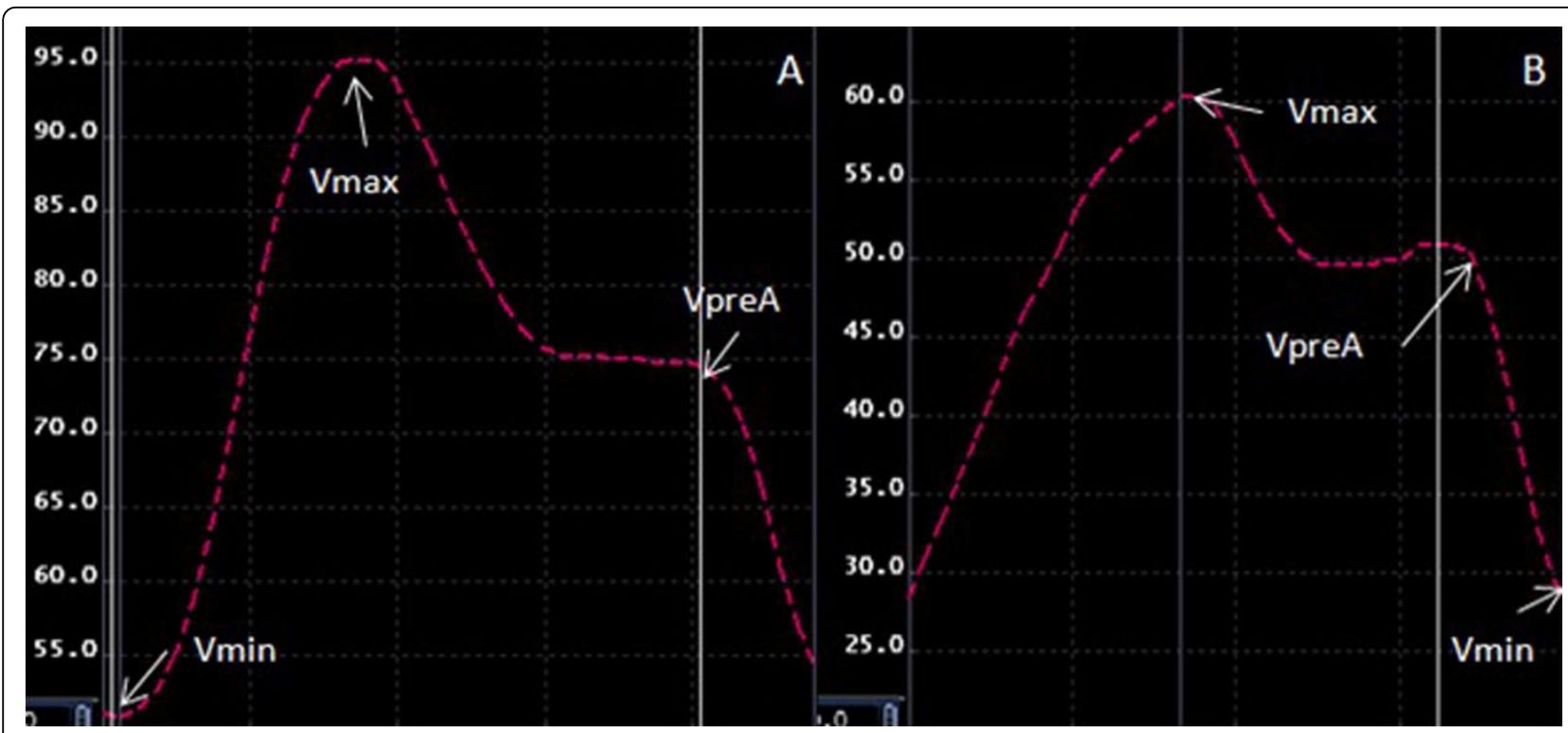

Figure 1 Multimodality Tissue Tracking, volume analysis. Subject with heart failure (Panel A) versus a healthy subject (Panel B).Vmax; maximal volume, VpreA; volume before pre-atrial contraction, Vmin; minimal volume.

Table 1 Comparison between the Simpson's method vs. Biplane method, Manual vs. MTT

\begin{tabular}{cccccc}
\hline \multicolumn{7}{c}{ Biplane } & Simpson's & $\mathbf{p}$ & $\mathbf{r}$ & $\mathbf{p}$ \\
\hline Manual & & & & \\
\hline Vmax (ml) & $84.3 \pm 34.6$ & $88.1 \pm 35.2$ & 0.08 & 0.95 & $<0.001$ \\
\hline LAEF (\%) & $0.44 \pm 0.1$ & $0.34 \pm 0.1$ & $<0.001$ & 0.88 & $<0.001$ \\
\hline LAPEF (\%) & $0.16 \pm 0.08$ & $0.11 \pm 0.05$ & $<0.001$ & 0.70 & $<0.001$ \\
\hline LAAEF(\%) & $0.37 \pm 0.07$ & $0.28 \pm 0.07$ & $<0.001$ & 0.70 & $<0.001$ \\
\hline MTT & & & & & \\
\hline Vmax (ml) & $86.5 \pm 33.6$ & $85.2 \pm 35.2$ & 0.53 & 0.95 & $<0.001$ \\
\hline LAEF (\%) & $0.46 \pm 0.2$ & $0.33 \pm 0.10$ & $<0.001$ & 0.85 & $<0.001$ \\
\hline LAPEF (\%) & $0.18 \pm 0.05$ & $0.11 \pm 0.04$ & $<0.001$ & 0.57 & 0.002 \\
\hline LAAEF(\%) & $0.38 \pm 0.08$ & $0.27 \pm 0.06$ & $<0.001$ & 0.61 & $<0.001$ \\
\hline \multicolumn{7}{c}{ Manual } & $p$ & $r$ & $p$ \\
\hline Biplane & & & & \\
\hline Vmax (ml) & $86.5 \pm 33$ & $84.4 \pm 34$ & 0.08 & 0.98 & $<0.001$ \\
\hline LAEF (\%) & $0.45 \pm 0.13$ & $0.44 \pm 0.12$ & 0.21 & 0.88 & $<0.001$ \\
\hline LAPEF (\%) & $0.17 \pm 0.05$ & $0.16 \pm 0.08$ & 0.67 & 0.57 & 0.002 \\
\hline LAAEF(\%) & $0.16 \pm 0.08$ & $0.36 \pm 0.09$ & 0.21 & 0.83 & $<0.001$ \\
\hline Simpson's & & & & & \\
\hline Vmax (ml) & $85.2 \pm 35.2$ & $88.2 \pm 35.2$ & $<0.001$ & 1.00 & $<0.001$ \\
\hline LAEF (\%) & $0.33 \pm 0.1$ & $0.34 \pm 0.1$ & 0.19 & 0.92 & $<0.001$ \\
\hline LAPEF (\%) & $0.11 \pm 0.04$ & $0.11 \pm 0.05$ & 0.43 & 0.29 & 0.14 \\
\hline LAAEF(\%) & $0.27 \pm 0.06$ & $0.28 \pm 0.06$ & 0.52 & 0.86 & $<0.001$ \\
\hline Results ar r & & & & \\
\hline
\end{tabular}

Results are reported as mean \pm standard deviation. Abbreviations: Vmax; maximal volume; LAEF, left atrial total ejection fraction; $L A P E F$, left atrial passive ejection fraction; LAAEF, left atrial active ejection fraction; Smax, maximal global strain (a longitudinal strain vs. b circumferential strain); $r$, Pearson's correlation.

\section{Funding}

NHLBI N01-HC-95159 NHLBI N01-HC-95168 NCRR UL1-RR-024156 NCRR UL1-RR-025005.

\section{Authors' details}

${ }^{1}$ Cardiovascular Imaging, Johns Hopkins University, Baltimore, Maryland, USA. ${ }^{2}$ Cardiology, University of Oslo, Oslo, Norway. ${ }^{3}$ National Institutes of Health, Bethesda, Maryland, USA. ${ }^{4}$ National Institutes of Health, London, UK.

Published: 16 January 2014

\section{doi:10.1186/1532-429X-16-S1-P348}

Cite this article as: Zareian et al: Left atrial structure and functional quantitation using cardiac magnetic resonance: comparison of manual delineation vs. multimodality tissue tracking based semi-automated methods. Journal of Cardiovascular Magnetic Resonance 2014 16(Suppl 1): P348.

\section{Submit your next manuscript to BioMed Central} and take full advantage of:

- Convenient online submission

- Thorough peer review

- No space constraints or color figure charges

- Immediate publication on acceptance

- Inclusion in PubMed, CAS, Scopus and Google Scholar

- Research which is freely available for redistribution 